\title{
Adherence to the Gluten-Free Diet during the Lockdown for COVID-19 Pandemic: A Web-Based Survey of Italian Subjects with Celiac Disease
}

\author{
Alice Monzani ${ }^{1, *(D)}$, Elena Lionetti ${ }^{2}$, Enrico Felici ${ }^{3}$, Lucia Fransos ${ }^{4}$, Danila Azzolina ${ }^{5}$, \\ Ivana Rabbone ${ }^{1}$ and Carlo Catassi ${ }^{2}$ \\ 1 Division of Pediatrics, Department of Health Sciences, Università del Piemonte Orientale, \\ 28100 Novara, Italy; ivana.rabbone@med.uniupo.it \\ 2 Department of Pediatrics, Marche Polytechnic University, 60020 Ancona, Italy; \\ m.e.lionetti@staff.univpm.it (E.L.); c.catassi@staff.univpm.it (C.C.) \\ 3 Pediatric and Pediatric Emergency Unit, Children Hospital, 15121 Alessandria, Italy; \\ enrico.felici@ospedale.al.it \\ 4 Piedmont Section, Italian Celiac Association, 10136 Turin, Italy; fransoslucia@hotmail.com \\ 5 Department of Translational Medicine, Unit of Medical Statistics and Cancer Epidemiology, \\ Università del Piemonte Orientale, 28100 Novara, Italy; danila.azzolina@uniupo.it \\ * Correspondence: alice.monzani@med.uniupo.it; Tel.: +39-03213733868; Fax: +39-03213733598
}

Received: 7 October 2020; Accepted: 9 November 2020; Published: 12 November 2020

\begin{abstract}
We aimed to assess the perceived impact of the lockdown, imposed to control the spreading of COVID-19, on the adherence of Italian celiac disease (CD) subjects to the gluten-free diet by a web-based survey. A total of 1983 responses were analyzed, 1614 (81.4\%) by CD adults and $369(18.6 \%)$ by parents/caregivers of $\mathrm{CD}$ children/adolescents. The compliance with the GFD was unchanged for $69 \%$ of the adults and $70 \%$ of the children, and improved for $29 \%$ of both. The factors increasing the probability to report stricter compliance were the presence of $\mathrm{CD}$ symptoms in the last year before the lockdown (odds ratio (OR) 1.98, 95\% confidence interval (CI) 1.46-2.26), a partial usual adherence to gluten-free diet (GFD) (OR 1.91, 95\% CI 1.2-3.06), and having tried recipes with naturally gluten-free ingredients more than usual (OR 1.58, 95\% CI 1.28-1.96) for adults; the presence of CD symptoms in the last year (OR 2.05, 95\% CI 1.21-3.47), still positive CD antibodies (OR 1.89, 95\% CI 1.14-3.13), and other family members with CD (OR 2.24, 95\% CI 1.3-3.85) for children/adolescents. Therefore, the lockdown led to a reported improved adherence to the GFD in one-third of the respondents, in particular in those with previous worse disease control, offering the opportunity to avoid sources of contamination/transgression and increase the use of naturally gluten-free products.
\end{abstract}

Keywords: celiac disease; gluten-free diet; COVID-19; coronavirus; lockdown; diet compliance; web survey; telemedicine

\section{Introduction}

In December 2019, a cluster of pneumonia cases caused by a novel coronavirus, named SARS-nCoV-2, occurred in Wuhan, China, and rapidly spread initially in Europe and later throughout the world. On 11 March, 2020, coronavirus disease 2019 (COVID-19) was declared a pandemic by the World Health Organization [1]. On 9 March, 2020, the Italian government imposed a national lockdown, and in the whole Country the same restrictive measures for the movements of the population were introduced with the aim of controlling the viral spreading. From then until 18 May, the Italian population was forced into an unprecedented homebound isolation. This has indeed impacted people's lifestyle and sometimes modified their quality of life. Such changes have been even more relevant 
for people with chronic diseases. Because of the COVID-19 pandemic, healthcare providers have abruptly changed their care delivery to protect patients and staff from infection and to reallocate the resource towards the greatest acute needs. Elective, routine, and nonemergency casework has stopped, and treating or supporting people with non-urgent and long-term conditions at a distance from healthcare providers by telemedicine and eHealth approaches has become imperative [2].

In the gastroenterological area, the impact of the lockdown on celiac disease (CD) has only limitedly been addressed [3]. CD has the peculiarity of merely dietetic treatment, which could be impacted in different ways by the restrictive measures, possibly implying difficulties in the supply of gluten-free products [3] or avoiding eating out, known to be an occasion of potential contamination or transgression to the GFD [4,5]. Previous findings on the impact of the lockdown on dietary habits in the general population are controversial, with some studies reporting a decrease in nutritional quality of diet on average worldwide [6-12], while a recent survey showed an increased healthy eating during the pandemic, thanks to less eating out and increased cooking [13]. Therefore, we aimed to assess the impact of the restrictive measures on the adherence of Italian CD subjects to the gluten-free diet (GFD) and on the management of the disease during the lockdown, by a web-based survey.

\section{Materials and Methods}

\subsection{Study Design}

We performed a cross-sectional survey, conducted in Italy during the lockdown by an online questionnaire, self-administered in Italian language, according to the Checklist for Reporting Results of Internet E-Surveys (CHERRIES) [14].

The probability sample is considered demographically representative of the whole population. An information sheet as the first page of the online survey was set, with participants required to check a box to indicate consent before accessing the survey. All the potential participants were fully informed about the study, the reason for conducting the research, how the data will be used, the extent of privacy, anonymity, and confidentiality, the voluntary nature of participating, information in case the respondents changed their mind during the survey, along with contact details for further information. The participation in the survey was voluntary and anonymous and took about 10-15 min. Confidentiality of the information was ensured, and no financial incentive to participate in the study was offered. The data were protected by a password which only the Principal Investigator had access to. The study was approved by the local Ethics Committee (CE 83/20).

\subsection{Survey Participants}

The web-based survey addressed both adult subjects (aged $\geq 18$ years) with a certified diagnosis of $\mathrm{CD}$ and parents/caregivers of children/adolescents (aged $<18$ years) with a certified diagnosis of $C D$.

\subsection{Survey Dissemination}

The Italian Celiac Society distributed to all members a link to the survey by their mailing list. The link to the web-based survey was posted also on chats through freely communicating apps (WhatsApp), and social networks (Instagram and Facebook), five weeks after the beginning of the lockdown (from 29 April to 1 June, 2020). Furthermore, snowball sampling, where existing study subjects recruit future subjects from among their acquaintances, was also used, as respondents were encouraged to pass it on to others. Individuals were directed via an electronic link to an online survey platform (Google Forms).

\subsection{Variables and Data Sources}

At the beginning of the survey, respondents had to select if they were either adults (aged $\geq 18$ years) with a certified diagnosis of celiac disease or parents/care givers of children/adolescents (aged $<18$ years) with a certified diagnosis of celiac disease. Thus, they were re-directed to the appropriate section. A single 
respondent could answer both the questionnaires for adult $C D$ subjects and for parents/caregivers of $\mathrm{CD}$ children/adolescents. At the end, respondents were able to review and change their answers.

The survey consisted of 33 items, distributed in 4 screens. There was a general part including sociodemographic questions (age, nationality, province of residence, educational level, housing conditions, self-perceived economic status, current employment status, and compliance with restrictive measures) and questions about CD diagnosis, followed by questions addressing different analysis domains (Supplementary Materials S1). The following analysis domains were explored: availability of gluten-free products, adherence to GFD, and access to care for CD during the lockdown.

Due to the lack of validated questionnaires about this specific topic, the survey was ad hoc designed to assess all the mentioned variables, consistently with the aim of the research. The questionnaire was tested in a sample of voluntary adult CD patients who reviewed the questionnaire individually and provided verbal feedback, also reporting the time needed to fill it.

\subsection{Study Endpoints}

The primary endpoint of this study was the perceived impact of the lockdown on the adherence to GFD of CD subjects. Secondary endpoints included difficulties in finding gluten-free products during the lockdown and the impact of the lockdown on CD management.

\subsection{Statistical Analysis}

Only completed questionnaires were analyzed. Data were summarized according to groups as median and interquartile range (IQR, 25th-75th percentile) and analyzed using the Wilcoxon test. Categorical variables, whenever dichotomous or nominal, were reported as frequencies and percentages and analyzed through the Chi-square test. Univariable analysis and multivariable proportional odds ordinal logistic regression were carried out to quantify the effects of the covariates on the different degrees of compliance to GFD. The estimated Odds Ratios (OR) together with the $95 \%$ confidence intervals have been reported.

The statistical analyses were conducted using R 3.5.2 [15] with the rms packages (Regression Modeling Strategies. R package version 4.1-3. 2014). The threshold of statistical significance was 0.05 for all tests used (two-tailed).

\section{Results}

\subsection{Study Population}

We received a total of 2021 responses; $19(0.9 \%)$ subjects did not give their consent to the interview, and $17(0.8 \%)$ were excluded because they declared not to be either adults with a certified diagnosis of CD or parents/caregivers of children/adolescents with CD. Therefore, 1983 responses were analyzed. Out of them, 1614 (81.4\%) were filled in by adults with CD and $369(18.6 \%)$ by parents/caregivers of children/adolescents with CD. The flow diagram of the survey is shown in Figure 1.

The response rate for all the items was higher than 93\%. Overall, respondents were from 103 out of 110 Italian provinces: 50\% from Northern Italy, 43\% from Central Italy, 7\% from Southern Italy. Among both adult subjects with CD and parents/caregivers of CD children, more than $99 \%$ were Italian (1607/1614 and 366/369, respectively). The median (IQR) age was 39 (28-50) years in the group of adults with CD and 45 (41-49) years in the group of parents/caregivers of CD children/adolescents. The median (IQR) age of the children whose parent/caregiver took part in the survey was 12 (7-14) years.

\subsection{Demographic and Socio-Economic Characteristics}

The baseline demographics and socio-economic features of the respondents are described in Table 1.

More than half of the respondents in both groups had an education degree lower than bachelors, the majority of the overall respondents were employed and were not going to the workplace during 
the lockdown. In most cases, their household members during the lockdown were unchanged, with no other family members with $\mathrm{CD}$ in about three-quarters of the respondents. In both groups, the compliance with the restrictive measures given by the Government about lockdown and social distancing was high or very high ( $97 \%$ in adults and $99 \%$ in parents/caregivers). Only $1 \%$ of the respondents were officially quarantined by the Authorities at the time of the survey. More than half of the respondents reported that the electronic devices in their possession were much or very much adequate to their needs, and about one-third of them considered their income much or very much adequate to their needs.

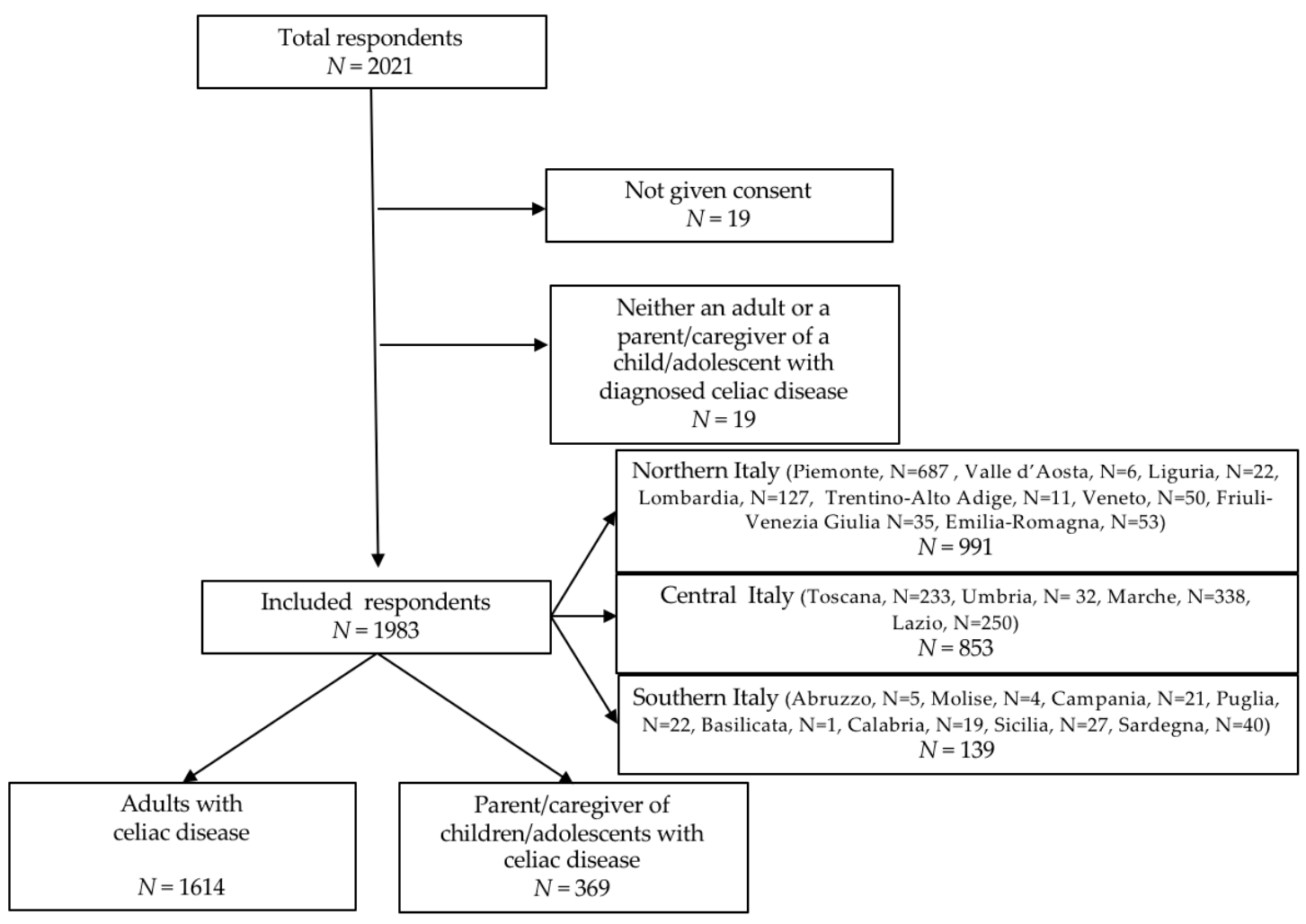

Figure 1. Flow diagram of the survey.

Table 1. Socio-demographic characteristics, working and housing conditions of the respondents.

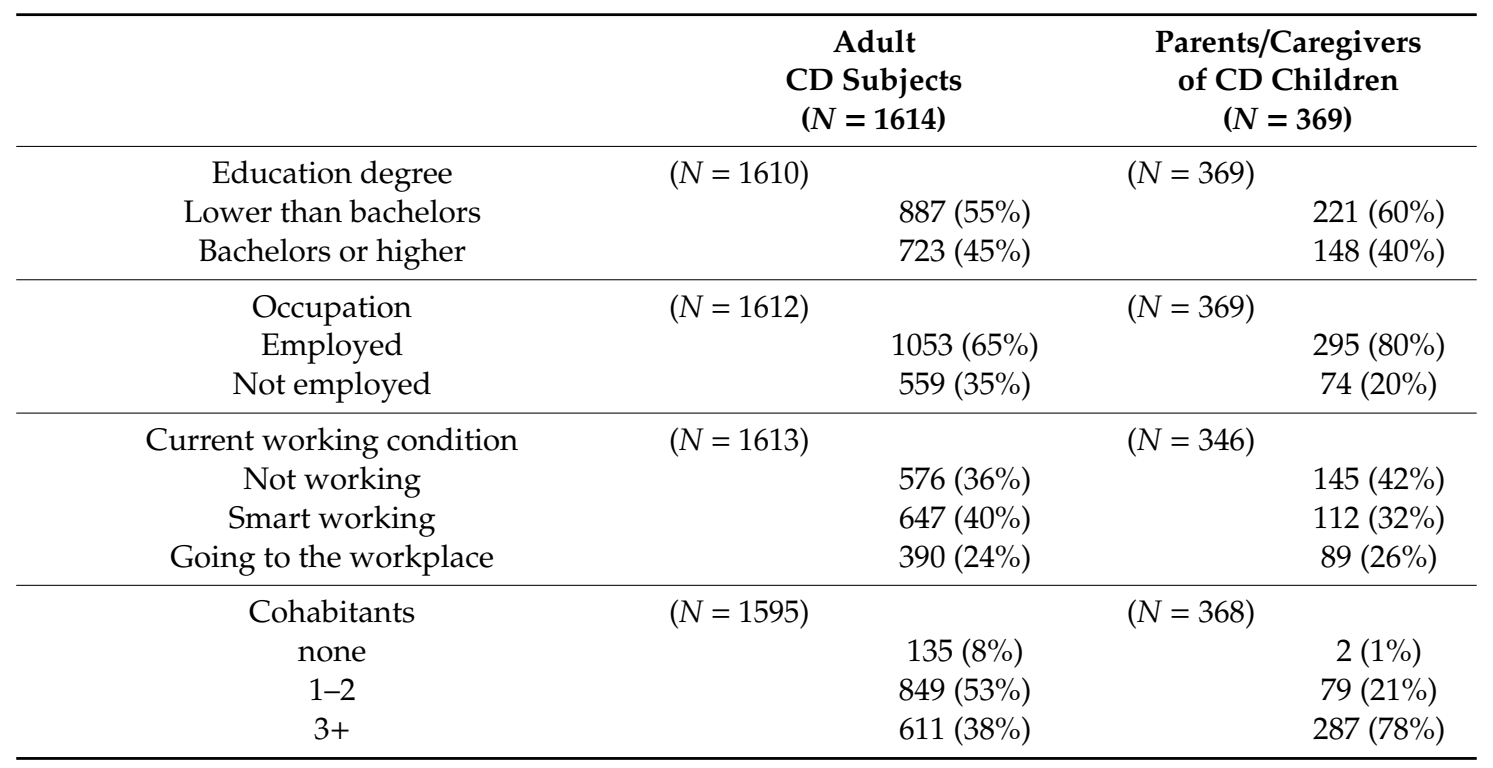


Table 1. Cont.

\begin{tabular}{|c|c|c|c|c|}
\hline & \multicolumn{2}{|c|}{$\begin{array}{c}\text { Adult } \\
\text { CD Subjects } \\
(N=1614)\end{array}$} & \multicolumn{2}{|c|}{$\begin{array}{c}\text { Parents/Caregivers } \\
\text { of CD Children } \\
(N=369)\end{array}$} \\
\hline Household members during the lockdown & $(N=1608)$ & & $(N=367)$ & \\
\hline Unchanged & & $1446(90 \%)$ & & $353(96 \%)$ \\
\hline Decreased & & $95(6 \%)$ & & $6(2 \%)$ \\
\hline Increased & & $67(4 \%)$ & & $8(2 \%)$ \\
\hline Other family members with CD & $(N=1614)$ & & $(N=369)$ & \\
\hline No & & $1246(77 \%)$ & & $291(79 \%)$ \\
\hline Yes & & $368(23 \%)$ & & $78(21 \%)$ \\
\hline Compliance with the restrictive measures & $(N=1614)$ & & $(N=369)$ & \\
\hline Much/very much & & $1559(97 \%)$ & & $367(99 \%)$ \\
\hline Not at all/little/enough & & $55(3 \%)$ & & $2(1 \%)$ \\
\hline Currently quarantined & $(N=1585)$ & & $(N=366)$ & \\
\hline No & & $1579(99 \%)$ & & $362(99 \%)$ \\
\hline Yes & & $14(1 \%)$ & & $4(1 \%)$ \\
\hline Housing condition & $(N=1605)$ & & $(N=367)$ & \\
\hline Big city downtown & & $1003(62 \%)$ & & $181(49 \%)$ \\
\hline Suburbs/Small-medium town-village & & $602(38 \%)$ & & $186(51 \%)$ \\
\hline Adequacy of electronic devices & $(N=1605)$ & & $(N=368)$ & \\
\hline Much/very much & & $1097(68 \%)$ & & $230(62.5 \%)$ \\
\hline Not at all/little/enough & & $508(32 \%)$ & & $138(37.5 \%)$ \\
\hline Adequacy of the income & $(N=1603)$ & & $(N=366)$ & \\
\hline Much/very much & & $552(34 \%)$ & & $103(28 \%)$ \\
\hline Not at all/little/enough & & $1051(66 \%)$ & & $243(72 \%)$ \\
\hline
\end{tabular}

\subsection{Diagnosis and Management}

The data about CD diagnosis and management are shown in Table 2.

Table 2. Data about CD diagnosis and management.

\begin{tabular}{|c|c|c|}
\hline & $\begin{array}{l}\text { Adult CD Subjects } \\
\qquad(N=1614)\end{array}$ & $\begin{array}{l}\text { CD Children } \\
(N=369)\end{array}$ \\
\hline Upper digestive tract endoscopy & $(N=1614)$ & $(N=369)$ \\
\hline Yes & $1579(98 \%)$ & $143(39 \%)$ \\
\hline No & $35(2 \%)$ & $226(61 \%)$ \\
\hline Symptoms at diagnosis & $(N=1614)$ & $(N=369)$ \\
\hline Yes & $1208(75 \%)$ & $269(73 \%)$ \\
\hline No & $406(25 \%)$ & $100(27 \%)$ \\
\hline Last $\mathrm{CD}$ antibodies negative & $(N=1614)$ & $(N=369)$ \\
\hline Yes & $1232(76 \%)$ & $241(65 \%)$ \\
\hline No & $382(24 \%)$ & $128(35 \%)$ \\
\hline CD symptoms in the last year & $(N=1614)$ & $(N=369)$ \\
\hline Yes & $532(33 \%)$ & $101(27 \%)$ \\
\hline No & $1082(67 \%)$ & $268(73 \%)$ \\
\hline Usual compliance with the GFD & $(N=1614)$ & $(N=369)$ \\
\hline Strict & $1524(94 \%)$ & $360(98 \%)$ \\
\hline Partial & $90(6 \%)$ & $9(2 \%)$ \\
\hline
\end{tabular}

Data are expressed as number and percentage of the responses for each item. CD: celiac disease; GFD: gluten-free diet. 
The median (IQR) age at CD diagnosis was 27 (16-39) years in adults and 5 (3-8) in children/adolescents, with a median (IQR) duration of CD of 11 (5-18) and 4 (1-8) years, respectively.

At diagnosis, an upper digestive tract endoscopy was performed in $89 \%$ of the adults and in $39 \%$ of the children/adolescents, and the diagnosis was made because of symptoms suggestive for CD in $75 \%$ and $73 \%$ of cases, respectively. At the last follow-up control, $76 \%$ of the adults and $65 \%$ of the children tested negative for CD antibodies. In the last year, before the COVID-19 pandemic, 33\% of the adults and $27 \%$ of the children had experienced symptoms deemed attributable to CD. As for the usual compliance with GFD, $94 \%$ of the CD adults and $98 \%$ of the parents/caregivers of CD children reported strict compliance.

\subsection{GFD during the Lockdown}

The data about the GFD during the lockdown are reported in Table 3.

Table 3. Data about GFD during the lockdown.

\begin{tabular}{|c|c|c|c|c|}
\hline & \multicolumn{2}{|c|}{$\begin{array}{c}\text { Adult } \\
\text { CD Subjects } \\
(N=1614)\end{array}$} & \multicolumn{2}{|c|}{$\begin{array}{l}\text { Parents/Caregivers } \\
\text { of CD Children } \\
(N=369)\end{array}$} \\
\hline Difficulties in finding GFD products & $(N=1614)$ & & $(N=369)$ & \\
\hline No & & $1088(67 \%)$ & & $267(72 \%)$ \\
\hline Yes & & $526(33 \%)$ & & $102(28 \%)$ \\
\hline Homemade recipes with naturally gluten-free ingredients & $(N=1614)$ & & $(N=369)$ & \\
\hline As usual & & $714(44 \%)$ & & $190(51 \%)$ \\
\hline More than usual & & $720(45 \%)$ & & $146(40 \%)$ \\
\hline Never & & $180(11 \%)$ & & $33(9 \%)$ \\
\hline Compliance with GFD during the lockdown & $(N=1614)$ & & $(N=369)$ & \\
\hline Stricter & & $469(29 \%)$ & & $108(29 \%)$ \\
\hline Less strict & & $29(2 \%)$ & & $3(1 \%)$ \\
\hline Unchanged & & $1116(69 \%)$ & & $258(70 \%)$ \\
\hline If stricter, for which reason $\S$ & $(N=469)$ & & $(N=108)$ & \\
\hline Not eating away from home & & $350(75 \%)$ & & $69(64 \%)$ \\
\hline More time to prepare food & & $188(40 \%)$ & & $46(43 \%)$ \\
\hline No social life/home alone & & $12(3 \%)$ & & n.a. \\
\hline Taking better care of myself/my child & & $72(15 \%)$ & & $10(9 \%)$ \\
\hline Higher control over my child & & n.a & & $28(26 \%)$ \\
\hline If less strict, for which reason $\S$ & $(N=29)$ & & $(N=3)$ & \\
\hline Less time to prepare food & & $1(3 \%)$ & & $0(0 \%)$ \\
\hline Boredom/sadness/worries & & $14(48 \%)$ & & $2(67 \%)$ \\
\hline More occasions for transgression & & $6(21 \%)$ & & $0(0 \%)$ \\
\hline Difficulties in finding gluten-free products & & $6(21 \%)$ & & $1(33 \%)$ \\
\hline
\end{tabular}

$\S$ more than one answer can be given to this question. CD: celiac disease; GFD: gluten-free diet; n.a.: not applicable. Data are expressed as number and percentage of the responses for each item.

About one-third of the respondents reported that they had difficulties in finding gluten-free commercially made products dispensed with the voucher during the lockdown period, without differences according to the housing condition (both among CD adults, 38\% of those living in suburbs/villages vs. $32 \%$ of those living in cities, $p=0.8$, and among parents of CD children, $30 \%$ of those living in suburbs/villages vs. $25 \%$ of those living in big cities, $p=0.2$ ). Moreover, $45 \%$ of the adults and $40 \%$ of the parents/caregivers reported to have increased the preparation of homemade recipes with naturally gluten-free ingredients (gluten-free cereals, meat, fish, vegetables, fruit, etc.). About $70 \%$ of the respondents declared their adherence to the GFD remained unchanged during the lockdown, but $29 \%$ of both adults and the parents of children reported an improved compliance, mainly due to not eating away from home and having more time to prepare food. No difference was found in the compliance with GFD according to the first or last weeks of the lockdown. 
In the adult group, no difference was found between younger and older subjects with respect to GFD compliance: assuming the median age as the cut-off, increased compliance was reported in 239 subjects $(30 \%)$ that were $<39$ year old and in 230 subjects $(28 \%)$ that were $\geq 39$ year old $(p=0.5)$.

\section{5. $C D$ Health Care Aspects}

The data about CD health care aspects during the lockdown are summarized in Table 4.

Table 4. Data about CD care aspects during the lockdown.

\begin{tabular}{|c|c|c|c|c|}
\hline \multirow{4}{*}{$\begin{array}{c}\text { CD symptoms during the lockdown } \\
\text { No } \\
\text { Yes }\end{array}$} & \multicolumn{2}{|c|}{$\begin{array}{l}\text { Adult CD Subjects } \\
\quad(N=1614)\end{array}$} & \multicolumn{2}{|c|}{$\begin{array}{l}\text { CD Children } \\
(N=369)\end{array}$} \\
\hline & $(N=1614)$ & & $(N=369)$ & \\
\hline & & $1356(84 \%)$ & & $333(90 \%)$ \\
\hline & & $258(16 \%)$ & & $36(10 \%)$ \\
\hline Consult for health care advice & $(N=1519)$ & & $(N=356)$ & \\
\hline Nobody, no need & & $1337(88 \%)$ & & $300(84 \%)$ \\
\hline Gastroenterologist & & $41(3 \%)$ & & $16(4 \%)$ \\
\hline Dietician/Nutritionist & & $12(1 \%)$ & & $2(1 \%)$ \\
\hline Family/Private doctor & & $76(5 \%)$ & & $30(8 \%)$ \\
\hline Patients association & & $29(2 \%)$ & & $4(1 \%)$ \\
\hline Friends with CD & & $24(1 \%)$ & & $4(1 \%)$ \\
\hline Mode for receiving health care advice & $(N=1502)$ & & $(N=352)$ & \\
\hline No need & & $1351(90 \%)$ & & $305(87 \%)$ \\
\hline Telephone or e-mail contact & & $139(9 \%)$ & & $47(13 \%)$ \\
\hline Outpatient visit & & $12(1 \%)$ & & $0(0 \%)$ \\
\hline Home visit & & $0(0 \%)$ & & $0(0 \%)$ \\
\hline Skipped CD appointments & $(N=1588)$ & & $(N=366)$ & \\
\hline No & & $1115(70 \%)$ & & $235(64 \%)$ \\
\hline Yes & & $473(30 \%)$ & & $131(36 \%)$ \\
\hline $\begin{array}{l}\text { Impact of the lockdown on CD } \\
\text { management }\end{array}$ & $(N=1614)$ & & $(N=369)$ & \\
\hline None & & $1182(73 \%)$ & & $289(78 \%)$ \\
\hline For the better & & $92(6 \%)$ & & $27(7 \%)$ \\
\hline For the worse & & $340(21 \%)$ & & $53(14 \%)$ \\
\hline
\end{tabular}

Data are expressed as number and percentage of the responses for each item. CD: celiac disease.

During the lockdown, only a small percentage of subjects $(16 \%$ of adults and $10 \%$ of children/adolescents) experienced symptoms attributable to $C D$, significantly lower than in the last year before the pandemic ( $p<0.001$ for both). Even if about one-third of subjects skipped any previously scheduled appointments related to CD during the lockdown, $90 \%$ of adults and $87 \%$ of parents/caregivers reported no need for consults for CD-related health care advice, and when needed, advices were provided by telephone or e-mail contact in almost all cases.

\subsection{Factors Influencing an Improved Adherence to GFD during Lockdown}

Answers from both $\mathrm{CD}$ adults and parents/caregivers of $\mathrm{CD}$ children were analyzed according to their reported adherence to GFD during the lockdown, and the significant ones are shown in Tables 5 and 6.

As shown in Table 5, in adult CD subjects, stricter compliance with GFD during the lockdown was more likely reported by those experiencing $\mathrm{CD}$ symptoms in the last year and during the lockdown, with a partial usual compliance to GFD, who tried homemade recipes with naturally gluten-free ingredients more than usual, and who reported the lockdown had impacted their management of CD for the better. Conversely, living with three or more cohabitants reduced the probability of stricter compliance during the lockdown. In the multivariable ordinal logistic regression model, only the 
presence of CD symptoms in the last year before the lockdown (OR 1.98, 95\% CI 1.46-2.26), a partial usual adherence to GFD (OR 1.91, 95\% CI 1.2-3.06), and having tried homemade recipes with naturally gluten-free ingredients more than usual (OR 1.58, 95\% CI 1.28-1.96) increased the probability to report stricter compliance during the lockdown.

Table 5. Factors influencing the adherence to GFD during the lockdown, as reported by adults with CD.

\begin{tabular}{|c|c|c|c|c|c|c|}
\hline Adherence to GFD & & Less Strict & Unchanged & Stricter & $\begin{array}{c}\text { Chi2 } \\
p \text {-Value }\end{array}$ & OR $(95 \% \mathrm{CI})$ \\
\hline \multirow{3}{*}{ Cohabitants } & 0 & $2(7 \%)$ & $86(8 \%)$ & $47(10 \%)$ & \multirow[t]{3}{*}{0.039} & $1.19(0.82-1.74)$ \\
\hline & $1-2$ & $11(38 \%)$ & $578(52 \%)$ & $160(56 \%)$ & & (REF) \\
\hline & $3+$ & $16(55 \%)$ & $440(40 \%)$ & $255(34 \%)$ & & $0.75(0.6-0.94)$ \\
\hline \multirow{2}{*}{ Last $\mathrm{CD}$ antibodies negative } & Yes & $14(48 \%)$ & $869(78 \%)$ & $349(74 \%)$ & \multirow[t]{2}{*}{$<0.001$} & (REF) \\
\hline & No & $15(52 \%)$ & $247(22 \%)$ & $120(26 \%)$ & & $1.05(0.82-1.35)$ \\
\hline \multirow{2}{*}{ CD symptoms in the last year } & No & $15(52 \%)$ & $804(72 \%)$ & $263(56 \%)$ & \multirow[t]{2}{*}{$<0.001$} & (REF) \\
\hline & Yes & $14(48 \%)$ & $312(28 \%)$ & $206(44 \%)$ & & $1.84(1.47-2.29)$ \\
\hline \multirow{2}{*}{ Usual compliance with the GFD } & Strict & $16(55 \%)$ & $1088(97 \%)$ & $420(90 \%)$ & \multirow[t]{2}{*}{$<0.001$} & (REF) \\
\hline & Partial & $13(45 \%)$ & $28(3 \%)$ & $49(10 \%)$ & & $2.28(1.44-3.6)$ \\
\hline \multirow{2}{*}{$\begin{array}{l}\text { Homemade recipes with } \\
\text { naturally gluten-free ingredients }\end{array}$} & More than usual & $15(52 \%)$ & $451(40 \%)$ & $254(54 \%)$ & \multirow[t]{2}{*}{$<0.001$} & $1.64(1.33-2.03)$ \\
\hline & As usual/Never & $14(48 \%)$ & $665(60 \%)$ & $215(46 \%)$ & & $(\mathrm{REF})$ \\
\hline \multirow{2}{*}{$\begin{array}{l}\text { CD symptoms during } \\
\text { the lockdown }\end{array}$} & No & $20(69 \%)$ & $961(86 \%)$ & $375(80 \%)$ & \multirow[t]{2}{*}{$<0.001$} & (REF) \\
\hline & Yes & $9(31 \%)$ & $155(14 \%)$ & $94(20 \%)$ & & $1.39(1.05-1.84)$ \\
\hline \multirow{3}{*}{$\begin{array}{l}\text { Impact of the lockdown on } \\
\text { CD management }\end{array}$} & None & $15(52 \%)$ & $871(78 \%)$ & $296(63 \%)$ & \multirow[t]{3}{*}{$<0.001$} & (REF) \\
\hline & For the better & $0(0 \%)$ & $21(2 \%)$ & $71(15 \%)$ & & $9.87(5.97-16.34)$ \\
\hline & For the worse & $14(48 \%)$ & $224(20 \%)$ & $102(22 \%)$ & & $1.15(0.88-1.5)$ \\
\hline
\end{tabular}

CD: celiac disease; GFD: gluten-free diet; REF: reference value. Total responses for the variable "Cohabitants":

$N=1595 ;$ for all the other reported variables: $N=1614$.

Table 6. Factors influencing the adherence to GFD during the lockdown, as reported by parents/caregivers of children with $\mathrm{CD}$.

\begin{tabular}{|c|c|c|c|c|c|c|}
\hline Adherence to GFD & & Less Strict & Unchanged & Stricter & $\begin{array}{c}\text { Chi2 } \\
p \text {-Value }\end{array}$ & OR $(95 \%$ CI $)$ \\
\hline Occupation & Employed & $2(76 \%)$ & $217(84 \%)$ & $76(70 \%)$ & 0.01 & (REF) \\
\hline \multirow{2}{*}{ Other family members with CD } & No & $3(100 \%)$ & $212(82 \%)$ & $76(70 \%)$ & \multirow[t]{2}{*}{0.028} & (REF) \\
\hline & Yes & $0(0 \%)$ & $46(18 \%)$ & $32(30 \%)$ & & $2(1.19-3.36)$ \\
\hline \multirow{2}{*}{ Last $\mathrm{CD}$ antibodies negative } & Yes & $0(0 \%)$ & $188(73 \%)$ & $53(49 \%)$ & \multirow[t]{2}{*}{$<0.001$} & (REF) \\
\hline & No & $3(100 \%)$ & $70(27 \%)$ & $55(51 \%)$ & & $2.44(1.54-3.87)$ \\
\hline \multirow{2}{*}{ CD symptoms in the last year } & No & $2(33 \%)$ & $203(79 \%)$ & $64(59 \%)$ & \multirow[t]{2}{*}{$<0.001$} & (REF) \\
\hline & Yes & $1(67 \%)$ & $55(21 \%)$ & $44(41 \%)$ & & $2.31(1.43-3.75)$ \\
\hline \multirow{2}{*}{$\begin{array}{l}\text { Impact of the lockdown on } \\
\text { CD management }\end{array}$} & For the better & $0(0 \%)$ & $10(4 \%)$ & $17(16 \%)$ & \multirow{2}{*}{$<0.001$} & $9.87(5.97-16.84)$ \\
\hline & For the worse & $1(33 \%)$ & $33(13 \%)$ & $19(18 \%)$ & & $1.15(0.88-1.5)$ \\
\hline
\end{tabular}

CD: celiac disease; REF: reference value. Total responses for all the reported variables: $N=369 .{ }^{\S}$ The median value of $C D$ duration was chosen as cut-off to convert the continuous variable "years since CD diagnosis" into a dichotomous variable.

As shown in Table 6, according to what was reported by parents/caregivers, CD children/adolescents who more likely improved their compliance with the GFD were those whose parents/caregivers were not employed during the lockdown, with other family members with $C D$, with a shorter duration of $C D$, whose last $C D$ antibodies tested positive, who experienced $C D$ symptoms during the last year and during the lockdown, and whose parents reported the lockdown had impacted the management of CD for the better. In the multivariable ordinal logistic regression model, the presence of CD symptoms in the last year before the lockdown (OR 2.05, 95\% CI 1.21-3.47), still positive CD antibodies (OR 1.89, 
95\% CI 1.14-3.13), and other family members with CD (OR 2.24, 95\% CI 1.3-3.85) increased the probability to report an improved compliance during the lockdown.

\section{Discussion}

Our survey is the first to explore the potential effects of the restrictive measure imposed during COVID-19 pandemic on the adherence to GFD and lifestyle of subjects with CD. Likely, CD does not represent itself a high-risk condition for the development of severe forms of COVID-19 disease and, therefore, CD patients had been reported not to feel more vulnerable because of their CD [3]. Nonetheless, it was foreseeable that lockdown may have impacted their lives differently from healthy subjects, due to both the limitations and the opportunities that the restrictive measures represented in the GFD perspective. Exploring the potential impact of this unprecedented home-bound isolation represents a unique opportunity to assess the management of the GFD in the absence of potential sources of contamination related to social life. In the general population, previous studies on the impact of the lockdown on dietary habits showed conflicting results. Some studies reported a negative worldwide impact on dietary habits by restrictive measures, promoting unhealthy behaviors in both children and adults [6-12]. Along with the increase in sedentary behaviors, higher odds for elevated frequency of ultraprocessed food consumption (sugary foods, snacks, ready-to-eat frozen foods, and embedded foods) and low consumption of fruit and vegetables were reported [6], resulting in a decrease in nutritional quality of diet on average [7]. However, it has also been reported that the intake of fast food and commercial pastries decreased, while consumption of homemade pastries increased, testifying a significant increase of the cooked-at-home preparations $[8,11]$. In our survey, the limitations imposed by the restrictive measures seem to have led subjects with $C D$ to virtuous dietary habits. A similar trend was described also by a recent online survey in healthy subjects, showing increased healthy eating during the pandemic, due to less eating out and increased cooking [13].

The most relevant result of our survey was that, despite facing a troublesome and dramatic global situation, almost one-third of the respondents reported a perceived improved adherence to GFD. In most of the subjects, the compliance with the GFD was unchanged compared with the previous period, with only $2 \%$ of the adults and $1 \%$ of the parents/caregivers reporting worsened compliance. When investigating the causes of the improved compliance, the most reported reason was not eating away from home, both in adults and children/adolescents. It is known that having meals in restaurants/cafes/canteens may represent a potential source of involuntary contamination [16]. Moreover, sharing the meal with non-CD diners could also be the occasion for voluntary transgressions, especially in adolescents $[4,5]$. Therefore, the forced isolation imposed by the restrictive measure represented an unprecedented condition for lowering the probability of both involuntary and voluntary contamination, leading to a perceived stricter compliance with GFD. Another reason for the improved compliance was reported to be the increased time available for food preparation. Accordingly, more than $40 \%$ of the respondents reported to have tried homemade recipes with naturally gluten-free ingredients more than usual, and this was associated with an improved compliance with GFD in adults. The increased cooking using naturally gluten-free ingredients goes hand-to-hand with the difficulties in finding commercially made GFD products, reported by about one-third of the respondents.

In the minimal percentage of subjects reporting a worse compliance with the GFD, the main reason seems to be the negative feelings related to the COVID-19 period, followed by difficulties in finding commercially made gluten-free products, reported by about one-third of the respondents. Therefore, the reduced compliance could be firstly attributable to depressive feelings and worries for the pandemic situation, and it could be hypothesized that the negative feelings related to the global situation lead someone to a "disinvestment" in his/her own health, resulting in behaviors (lower attention to possible contamination or voluntary transgressions) leading to a worse compliance to the GFD. Moreover, it should be noted that, in Italy, gluten-free products are supplied to patients for free with a voucher only within their region of residence, and the restrictions for people's movements may have represented a substantial obstacle. In a recent Italian survey, about half of the CD respondents 
reported to be a little to very much worried about the possible shortness of gluten-free food at the beginning of the pandemic [3]. Along with the difficulties in finding commercially made gluten-free products, the lockdown could have given some CD subjects the chance to discover more possibilities of a strict GFD based on naturally gluten-free ingredients.

When analyzing in detail which subjects had a higher probability to report stricter compliance during the lockdown, they turned out to be those with CD-attributable symptoms both before and during the lockdown, suggesting a worse disease control. In adults, this inference was supported by the evidence that the reported compliance was improved in those who had only a partial compliance before the pandemic. It could be assumed that a "beneficial" effect of the isolation was higher just in those who were less compliant with the dietetic treatment and that the presence of symptoms prompted them to pay more attention to contamination/transgressions. Actually, the overall prevalence of subjects reporting CD-related symptoms decreased during the lockdown compared with the last year, and the stricter adherence to the GFD experienced during the pandemic by some subjects would possibly lead to a reduction of their symptoms in the following months. Interestingly, both adults and parents/caregivers reporting a higher compliance declared that the lockdown positively impacted their CD management, highlighting the perceived adherence to the GFD as a key element for a satisfactory management of CD.

In children and adolescents, an improved adherence to the GFD was reported when the parents/caregivers were not employed, likely having more time for food preparation, and when there were other family members with CD, suggesting a higher familiarity with the GFD. Children with a more recent diagnosis, especially those with still positive CD antibodies, were more likely to improve their compliance with the GFD.

In addition to the effects regarding the adherence to the GFD, the restrictive measures had an impact on CD management and access to health care consultations. In our sample, most of the respondents did not need consultations for health care advice. This might be explained by the fact that the prevalence of subjects experiencing CD-attributable symptoms significantly decreased during the lockdown. It might be hypothesized that the subjects followed stricter adherence to GFD so as to prevent potential exposure to COVID-19 during hospital/doctor visits if they had disease flare ups due to dietary transgressions. When needed, the most frequently reported way to receive health care advice was remote consultation by telephone or email with family doctors, private doctors, or gastroenterologists. The raising of telemedicine for the management of CD patients during the COVID-19 pandemic has recently been assessed by a two-center Italian survey, pointing out an overall satisfaction of CD subjects for this approach for CD healthcare [3].

The strengths of our study are as follows: (a) it is the first to report on the perception of CD subjects about the impact of the COVID-19-related restrictive measures on the adherence to the GFD; (b) it addresses both CD adults and parents/caregivers of CD children/adolescents; (c) the web-based method gave us the opportunity to reach a geographically dislocated population during a short time. Our study also has some limitations: (a) the lack of use of validated questionnaires; (b) that only the personal perceptions of the respondents are presented, as an intrinsic limit of the surveys. In this specific case, the improved, unchanged, or worsened compliance with the GFD reported by respondents should be verified by $C D$ antibody tests performed after the end of the lockdown, compared with previous ones. Moreover, in the outpatient visits performed after the lockdown it would be interesting to evaluate possible changes in body weight or composition, and to correlate them with lifestyle and dietary habits occurred during the lockdown.

\section{Conclusions}

In conclusion, the global impact of the lockdown on CD was not negative, being the compliance to GFD unchanged for $70 \%$ of the respondents, and even improved for $29 \%$, in particular for those with a previous worse disease control, due to reduced occasions for contamination/transgression and increased use of naturally gluten-free ingredients. It is desirable that the positive aspects of dietary 
management emerged during the lockdown would be maintained also in the future. Nutritionists and gastroenterologists dealing with CD patients should be aware of the virtuous behaviors that have been implemented in this critical period, such as the greater use of naturally gluten-free products, to promote them also in everyday life.

Supplementary Materials: The following are available online at http://www.mdpi.com/2072-6643/12/11/3467/s1, Questionnaires S1: questionnaires for adult CD subjects and parents/caregivers of CD children.

Author Contributions: Conceptualization, A.M., E.F. and L.F.; data curation, A.M., E.L. and D.A.; formal analysis, D.A.; investigation, A.M., E.L. and L.F.; methodology, A.M. and D.A.; resources, E.L.; supervision, E.F., I.R. and C.C.; writing - original draft, A.M.; writing—review and editing, E.L., E.F., L.F., D.A., I.R. and C.C. All authors have read and agreed to the published version of the manuscript.

Funding: This research received no external funding.

Acknowledgments: We thank the SIGENP CD-working group, the national Italian Celiac Society and its Piedmont, Marche, and Sardinia sections for their help in spreading the survey.

Conflicts of Interest: The authors declare no conflict of interest.

\section{References}

1. World Health Organization. Coronavirus Disease 2019 (COVID-19). Situation Report-74. Updated 11 March 2020. Available online: https://www.who.int/docs/default-source/coronaviruse/situation-reports/ 20200311-sitrep-51-covid-19.pdf?sfvrsn=1ba62e57_10 (accessed on 18 June 2020).

2. Berg, E.A.; Picoraro, J.A.; Miller, S.D.; Srinath, A.; Franciosi, J.P.; Hayes, C.E.; Farrell, P.R.; Cole, C.R.; LeLeiko, N.S. COVID-19-A Guide to Rapid Implementation of Telehealth Services: A Playbook for the Pediatric Gastroenterologist. J. Pediatr. Gastroenterol. Nutr. 2020, 70, 734-740. [CrossRef] [PubMed]

3. Siniscalchi, M.; Zingone, F.; Savarino, E.V.; D'Odorico, A.; Ciacci, C. COVID-19 pandemic perception in adults with celiac disease: An impulse to implement the use of telemedicine: COVID-19 and CeD. Dig. Liver Dis. 2020, 52, 1071-1075. [CrossRef] [PubMed]

4. White, L.E.; Bannerman, E.; Gillett, P.M. Coeliac disease and the gluten-free diet: A review of the burdens; factors associated with adherence and impact on health-related quality of life, with specific focus on adolescence. J. Hum. Nutr. Diet. 2016, 29, 593-606. [CrossRef] [PubMed]

5. Czaja-Bulsa, G.; Bulsa, M. Adherence to Gluten-Free Diet in Children with Celiac Disease. Nutrients 2018, 10, 1424. [CrossRef] [PubMed]

6. Werneck, A.O.; Silva, D.R.; Malta, D.C.; Gomes, C.S.; Souza-Júnior, P.R.B.; Azevedo, L.O.; Barros, M.B.A.; Szwarcwald, C.L. Associations of sedentary behaviors and incidence of unhealthy diet during the COVID-19 quarantine in Brazil. Public Health Nutr. 2020, 22, 1-12. [CrossRef] [PubMed]

7. Marty, L.; de Lauzon-Guillain, B.; Labesse, M.; Nicklaus, S. Food choice motives and the nutritional quality of diet during the COVID-19 lockdown in France. Appetite 2020, 157, 105005. [CrossRef] [PubMed]

8. Kriaucioniene, V.; Bagdonaviciene, L.; Rodríguez-Pérez, C.; Petkeviciene, J. Associations between Changes in Health Behaviours and Body Weight during the COVID-19 Quarantine in Lithuania: The Lithuanian COVIDiet Study. Nutrients 2020, 12, 3119. [CrossRef] [PubMed]

9. Błaszczyk-Bębenek, E.; Jagielski, P.; Bolesławska, I.; Jagielska, A.; Nitsch-Osuch, A.; Kawalec, P. Nutrition Behaviors in Polish Adults before and during COVID-19 Lockdown. Nutrients 2020, 12, 3084. [CrossRef] [PubMed]

10. Robinson, E.; Boyland, E.; Chisholm, A.; Harrold, J.; Maloney, N.G.; Marty, L.; Mead, B.R.; Noonan, R.; Hardman, C.A. Obesity, eating behavior and physical activity during COVID-19 lockdown: A study of UK adults. Appetite 2020, Sep 3, 104853. [CrossRef]

11. Carroll, N.; Sadowski, A.; Laila, A.; Hruska, V.; Nixon, M.; Ma, D.W.L.; Haines, J.; On Behalf of The Guelph Family Health Study. The Impact of COVID-19 on Health Behavior, Stress, Financial and Food Security among Middle to High Income Canadian Families with Young Children. Nutrients 2020, 12, 2352. [CrossRef] [PubMed]

12. Pietrobelli, A.; Pecoraro, L.; Ferruzzi, A.; Heo, M.; Faith, M.; Zoller, T.; Antoniazzi, F.; Piacentini, G.; Fearnbach, S.N.; Heymsfield, S.B. Effects of COVID-19 Lockdown on Lifestyle Behaviors in Children with Obesity Living in Verona, Italy: A Longitudinal Study. Obesity 2020, 28, 1382-1385. [CrossRef] [PubMed] 
13. Flanagan, E.W.; Beyl, R.A.; Fearnbach, S.N.; Altazan, A.D.; Martin, C.K.; Redman, L.M. The impact of COVID-19 stay-at-home orders on health behaviors in adults. Obesity 2020. [CrossRef]

14. Eysenbach, G. Improving the quality of web surveys: The Checklist for Reporting Results of Internet E-Surveys (CHERRIES). J. Med. Internet Res. 2004, 6, e34. [CrossRef]

15. R Core Team. R: A Language and Environment for Statistical Computing; R Foundation for Statistical Computing: Vienna, Austria, 2015.

16. Lerner, B.A.; Phan Vo, L.T.; Yates, S.; Rundle, A.G.; Green, P.H.R.; Lebwohl, B. Detection of Gluten in Gluten-Free Labeled Restaurant Food: Analysis of Crowd-Sourced Data. Am. J. Gastroenterol. 2019, 114, 792-797. [CrossRef] [PubMed]

Publisher's Note: MDPI stays neutral with regard to jurisdictional claims in published maps and institutional affiliations.

(C) 2020 by the authors. Licensee MDPI, Basel, Switzerland. This article is an open access article distributed under the terms and conditions of the Creative Commons Attribution (CC BY) license (http://creativecommons.org/licenses/by/4.0/). 